\title{
Acute calcific tendinitis of the longus colli muscle: case report and review of the literature
}

\author{
A. H. Zibis • D. Giannis $\cdot$ K. N. Malizos • \\ P. Kitsioulis • D. L. Arvanitis
}

Received: 26 May 2012/Revised: 26 October 2012/ Accepted: 7 November 2012/Published online: 21 November 2012

(C) Springer-Verlag Berlin Heidelberg 2012

\begin{abstract}
Purpose Acute calcific tendinitis of the longus colli muscle (or retropharyngeal tendinitis) is an aseptic inflammatory process characterized by acute posterior neck pain, neck stiffness and dysphagia or odynophagia. Awareness of its existence is crucial in the differential diagnosis, because many other conditions, such as retropharyngeal abscess, meningitis or disc herniation, show similar clinical features. We present a case exhibiting an uncommon symptom (torticollis) and a brief literature review to emphasize the risk of misdiagnosis.

Methods A 36-year-old woman presented with neck stiffness and torticollis accompanied by dysphagia and prevertebral space sensitivity on the second day.

Results The diagnosis was established by computed tomography (CT), the gold standard for identifying the presence of prevertebral oedema and calcific deposition associated with retropharyngeal tendinitis. Treatment with NSAIDs and low doses of corticosteroids relieved the symptoms within $48 \mathrm{~h}$.
\end{abstract}

A. H. Zibis $(\bowtie)$ D. L. Arvanitis

Department of Anatomy, Medical School,

University of Thessalia, Neofytoy 9 St.,

41223 Larissa, Greece

e-mail: ahzibis@gmail.com

D. Giannis

Medical School, University of Thessalia, Larissa, Greece

K. N. Malizos

Department of Orthopaedics, Medical School,

University of Thessalia, Larissa, Greece

P. Kitsioulis

Department of Anatomy, Medical School,

University of Ioannina, Ioannina, Greece
Conclusions Retropharyngeal tendinitis is an underreported entity in the literature and orthopaedists should become aware of its existence. Misdiagnosis of this important mimicker may lead to unnecessary antibiotics administration and interventions in the retropharyngeal space.

Keywords Acute calcific tendinitis of the longus colli . Retropharyngeal tendinitis - Torticollis · Prevertebral oedema - Dysphagia

\section{Introduction}

Acute calcific tendinitis of the longus colli muscle is an aseptic inflammatory process located in the cervical prevertebral space. The proposed pathogenesis of this entity is the deposition of calcium hydroxyapatite crystals at the $\mathrm{C} 1-\mathrm{C} 3$ vertebral level. Ignorance of its existence could result in additional discomfort of the patient and unnecessary surgical or pharmaceutical interventions, because it can be easily misdiagnosed as a retropharyngeal abscess. CT is the gold standard for identifying the presence of prevertebral oedema and calcific deposition, two determinant findings in establishing the diagnosis of retropharyngeal tendinitis. Relief of symptoms can be easily achieved by the use of NSAIDs, corticosteroids and cervical immobilization. We report a case exhibiting an uncommon symptom (torticollis) and a brief review of literature.

\section{Case report}

A 36-year-old woman presented with torticollis and a 12-h history of neck stiffness. Imaging control with plain radiography did not show any pathological signs. The patient was treated with a soft cervical collar, NSAIDs and muscle 
relaxants. On the second day of treatment the symptoms exacerbated. After revaluation it was decided to increase the dosage of muscle relaxants. Later that day, she reported further exacerbation of symptoms and dysphagia (for both liquids and solids) with corresponding local sensitivity in cervical prevertebral tissue while the patient reported general malaise. Cervical face and profile X-rays were performed without specific finding (Fig. 1). The haematological analysis showed mild increase of erythrocyte sedimentation rate $(\mathrm{ESR}=25 \mathrm{~mm} / \mathrm{h})$ and C-reactive protein $(\mathrm{CRP}=1.8 \mathrm{mg} /$ dl) accompanied by low grade pyrexia (up to $37.7^{\circ} \mathrm{C}$ ). Given the clinical presentation and examination findings, a cervical MRI was performed without using contrast medium (due to history of strong allergic reactions). Despite revealing an extended prevertebral oedema, it was not possible to distinguish the presence of abscess (Fig. 2). The patient was referred to an otorhinolaryngology clinic to investigate the existence of retropharyngeal abscess. A cervical CT was performed with contrast medium after prophylaxis with corticosteroids, but it did not reveal any abscess. Nevertheless, a focus of multiple small punctate calcific deposits became apparent at the $\mathrm{C} 1-\mathrm{C} 3$ vertebral level (Fig. 3). Considering the clinical presentation and after review of the literature, the patient was diagnosed with acute calcific tendinitis of the longus colli muscle and was treated with NSAIDs, low doses of corticosteroids and use of soft cervical collar. The symptoms began to resolve within approximately $12 \mathrm{~h}$ from the initiation of therapy and full range of neck motion was recovered within $48 \mathrm{~h}$. Fifteen months later, the patient is symptom-free.

\section{Discussion}

The longus colli muscle is located in the prevertebral area and consists of three parts: upper oblique, vertical and lower oblique fibers (Fig. 4). Upper oblique fibers arise from the anterior tubercles of the transverse processes of C3-C5 vertebrae to the anterior tubercle of atlas, vertical fibers extend from the bodies of the upper thoracic and lower cervical vertebrae to the bodies of the upper cervical vertebrae and lower oblique fibers extend from the front part of the T1-T3 vertebral bodies to the anterior tubercles of the transverse processes of C5-C6 vertebrae.

Acute calcific tendinitis of the longus colli muscle is an inflammatory process affecting the upper oblique fibers. The most common characteristic triad of symptoms for this disease consists of acute neck pain, neck stiffness and odynophagia [1, 2]. In addition, the haematological analysis may show mild elevation of erythrocyte sedimentation rate (ESR) accompanied by low grade pyrexia and mild leukocytosis [1, 3, 5, 10]. Nevertheless, several other symptoms and clinical findings may be associated with this entity such as dysphagia, sore throat, limited range of neck motion, pharyngeal oedema, erythema of nasopharynx and neck spasm [1, 3].

The presentation is not always typical, considering that, as we stated previously, many symptoms are associated with this disease. Our patient initially did not have the classic triad, but she presented with neck stiffness and torticollis, an unusual symptom in the literature. Later in the course of the illness, dysphagia and local sensitivity completed the clinical picture and guided us to consider acute calcific tendinitis of the longus colli as a possible diagnosis.

The exact aetiology of calcium hydroxyapatite crystals deposition is not wellknown, but some authors hypothesize that repetitive trauma, ischaemia, necrosis and tendinous degeneration play a role in the pathogenesis of retropharyngeal tendinitis $[3,5,6]$. The degenerative calcification hypothesis was supported by Mohr and Bilger describing calcific tendinopathies as a result of tendon cells necrosis and subsequent intracellular calcium accumulation [13].
Fig. 1 Cervical spine plan $\mathrm{X}$-rays, face (a) and profile (b), no specific findings were noticed
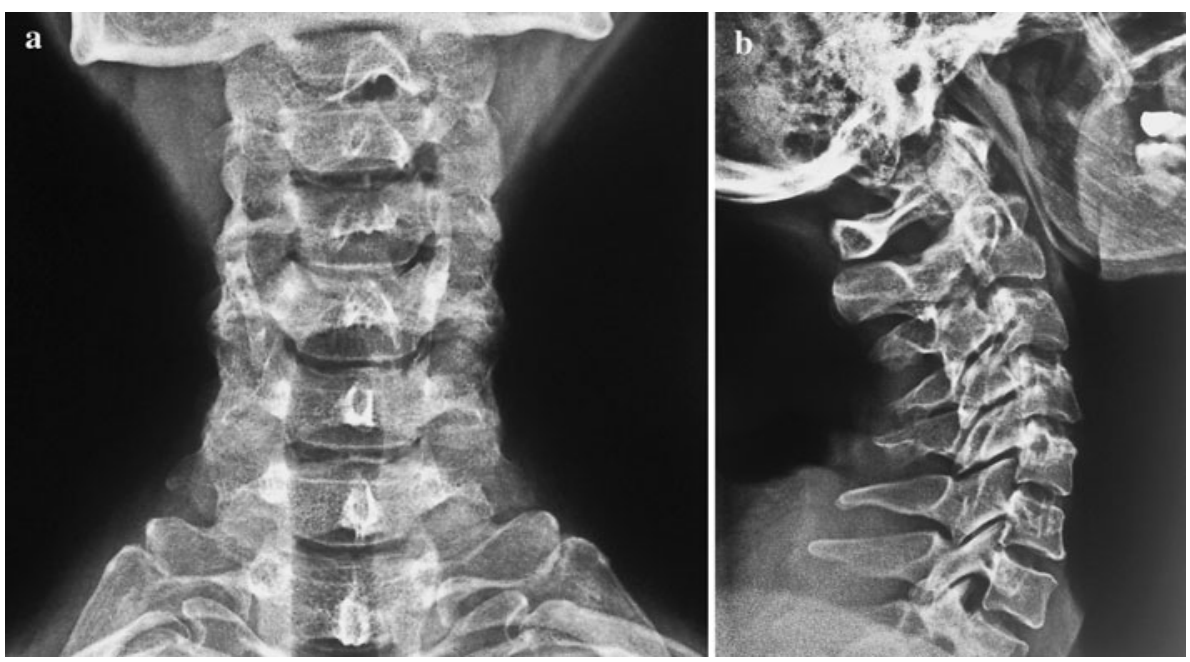

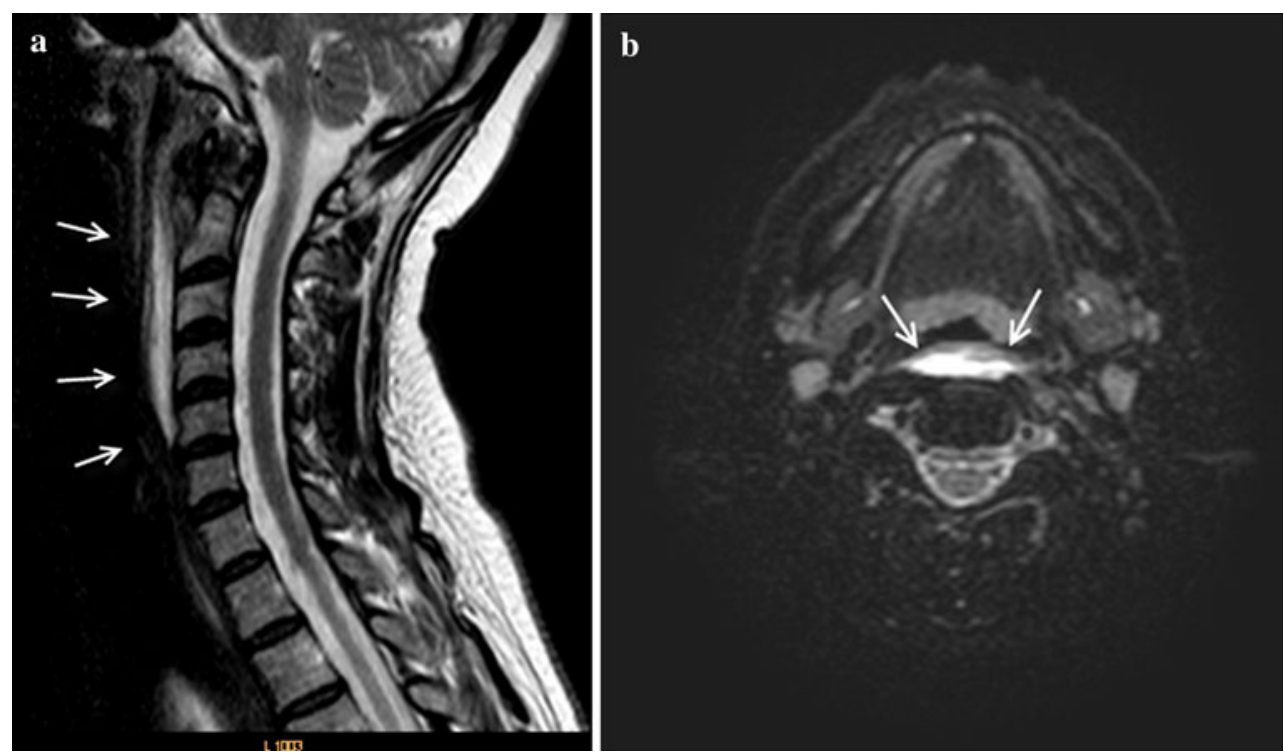

Fig. 2 a T2 W TSE sagittal view. b B-FFE transverse view. Both of them reveal an extended prevertebral oedema (arrows) without finding of abscess
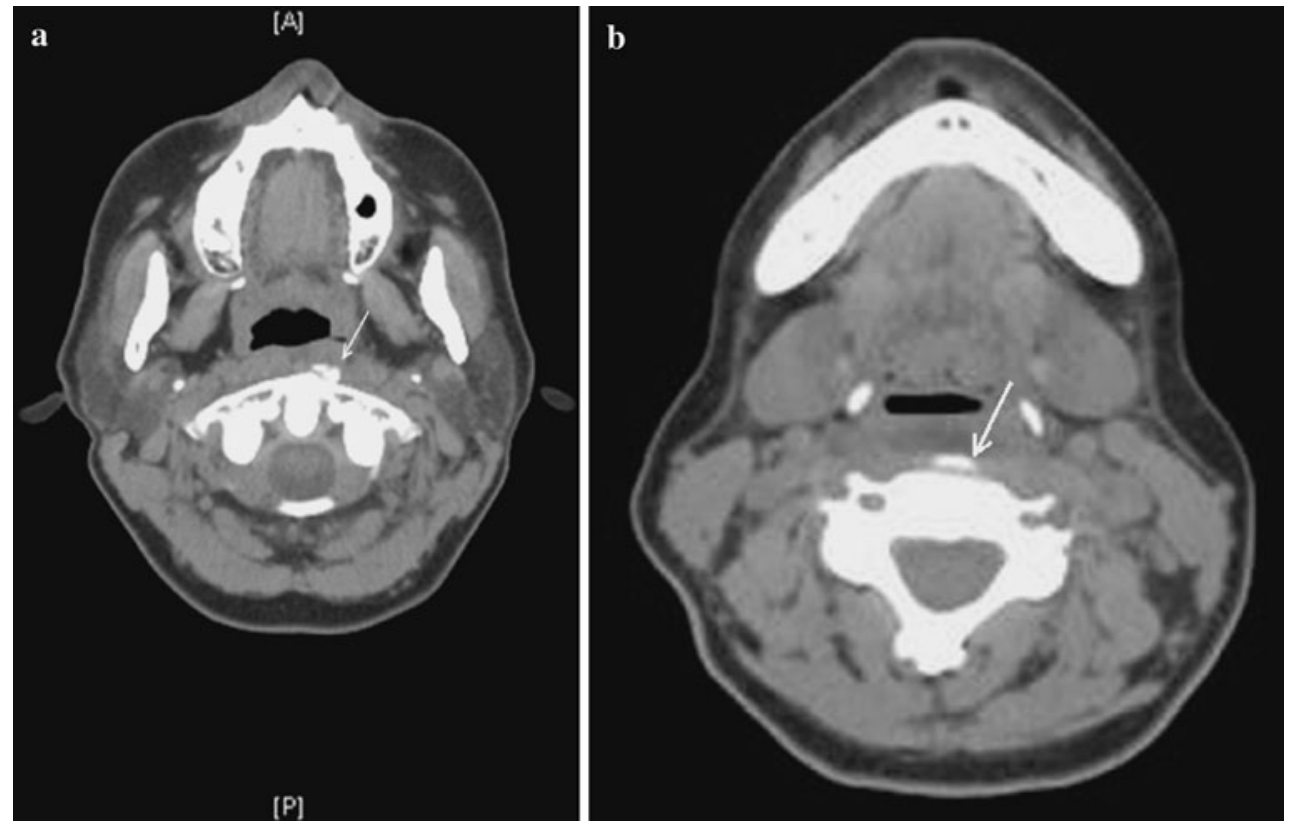

Fig. 3 Cervical CT on C1-C2 level (a) and C3 level (b) reveal multiple small punctate calcific deposits (arrows) in the mass of longus colli muscle

However, clinical and preclinical studies have definitely shown that calcification responsible for calcific tendinopathies is the result of an active cell-mediated process [13-15]. Uhthoff and Loehr [15] proposed that the natural course of this entity is divided into five phases: precalcific, formative, resting, resorptive and postcalcific. The precalcific phase is characterized by fibrocartilaginous metaplasia of tendon cells (triggered by an unknown factor) accompanied by accumulation of calcium crystals into the matrix vesicles (small extracellular organelles inhibited from mineralizing in normal tendon extracellular matrix, but involved in mineralization of the extracellular matrix of other tissues, such as bone and cartilage). The formation of calcium foci separated by fibrocartilage cells (formative phase) is followed by the resting phase. During this process, the multifocal calcium deposition presents, surrounded by the metaplastic fibrocartilage cells. Subsequently, the appearance of vascular channels and multinucleated cells/macrophages-mediated debris phagocytosis (resorptive phase) precedes the postcalcific phase that 


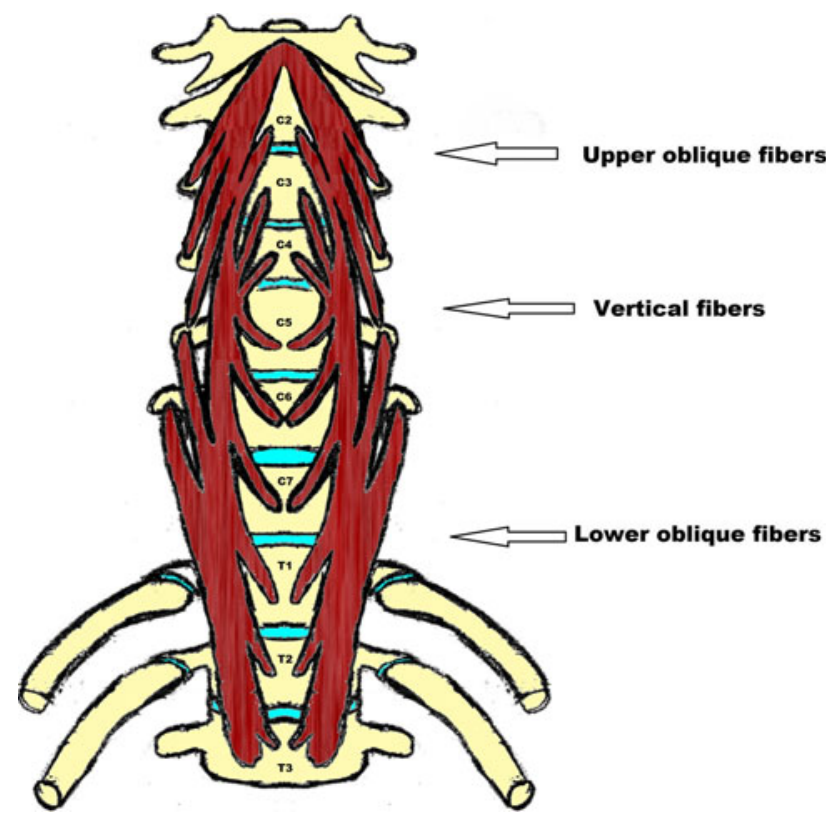

Fig. 4 Schematic illustration of the three parts of longus colli muscle

involves fibroblast-mediated deposition of type III collagen, resulting in the formation of granulomatous tissue. Benjamin et al. proposed that endochondral ossification is the main pathogenetic mechanism of Achilles tendon calcific tendinopathy [13]. According to their model, fibrocartilaginous metaplasia is the initiating event followed by erosion of calcaneal cartilage and invasion of blood vessels into the fibrocartilage metaplastic tissue [13]. Finally, ossification occurs and the calcific deposition is formed. Recently, Rui et al. suggested that erroneous differentiation of tendon-derived stem cells (TDSC) into chondrocytes or osteoblasts may be the possible mechanism leading to calcific tendinopathy $[13,14]$. The causes leading to erroneous differentiation of TDSC's is not clear at the moment, but both biological and mechanical factors are supposed to contribute [14]. Rupture of these crystals causes an aseptic foreign-body type inflammatory response and results in formation of reactive fluid in the retropharyngeal space surrounding the muscle $[3,4]$.

Usual imaging control consists of CT scan, MRI and plain radiography. CT scan is the gold standard for diagnosing this condition as it can detect both the prevertebral oedema and calcium hydroxyapatite crystals deposition at the longus colli tendon $[1,4,9]$. Furthermore, CT is considered the most sensitive imaging technique for distinguishing retropharyngeal tendinitis from retropharyngeal abscess [3, 4]. MRI can show the prevertebral oedema and corresponding fluid effusion, whereas it is difficult to recognize the calcific deposits with this imaging method $[1,4$, $7,9]$. Plain radiography may miss the calcareous deposition and the prevertebral soft-tissue swelling, since the oedema rapidly disappears, or may not show any pathological signs $[7,11]$.

The true incidence of the disease is still unknown [6]. In an era, when radiograph was the only radiological examination available for patients presenting with symptoms located in the head-neck region, while the use of CT was limited, the diagnosis of acute calcific tendinitis of the longus colli was probably missed [6]. Nowadays the definitive diagnosis is established by the use of CT and thus the true incidence is considered to be higher than previously estimated [4].

Through our PubMed search, we have concluded that only four publications correspond to orthopaedic journals. Orthopaedists should be aware of this underrecognized entity and its typical radiological findings to avoid diagnostic traps. Lack of knowledge could result in misdiagnosis and unnecessary interventions such as antibiotics administration or incision and drainage of the retropharyngeal space, especially if retropharyngeal abscess is suspected. Subsequently, the patient feels undue discomfort and the discharge from hospital is delayed.

Differential diagnosis between retropharyngeal tendinitis and other diseases showing similar clinical features, such as meningitis, retropharyngeal abscess, neoplasm, cervical disc herniation and fracture dislocation, is considered critical to avoid unnecessary interventions $[1,7,8]$. The most important mimicker of longus colli tendinitis is retropharyngeal abscess $[1,5,9]$. Despite showing the same clinical presentation, these two entities can be differentiated with a CT scan, the most appropriate method to reveal the characteristic calcareous deposition at the C1-C3 vertebral level [3, 4].

Acute calcific tendinitis of the longus colli muscle tends to be a self-limiting disease $[1,3,5,6]$. Non-steroidal antiinflammatory drugs (NSAIDs) are the first-line treatment, but in severe cases additional drugs, like corticosteroids or opioids, are suggested [1, 3, 7, 8]. Immobilization with soft cervical collar is another useful method to avoid aggravation of symptoms [1]. The symptoms, usually, begin to resolve within a couple of days from the initiation of treatment and the patients become symptom-free after $1-3$ weeks $[3,5,10-12]$.

\section{Conclusion}

Acute calcific tendinitis of the longus colli muscle is an unusual clinical entity and the bibliographic reference is limited. Nevertheless it is estimated that the real incidence is higher, considering the fact that the diagnosis is based on the clinical image and imaging investigation with cervical CT. Knowledge of the existence of this underreported disease, as well as awareness of its characteristic 
radiological appearance, is considered particularly useful for the clinician so as to avoid diagnostic errors, between entities such as torticollis and retropharyngeal abscess.

Conflict of interest None of the authors has any potential conflict of interest.

\section{References}

1. Ring D, Vaccaro AR, Scuderi G, Pathria MN, Garfin SR (1994) Acute calcific retropharyngeal tendinitis. Clinical presentation and pathological characterization. J Bone Joint Surg Am 76(11):1636-1642

2. Hviid C, Salomonsen M, Gelineck J, Rasmussen LR, Jensen KM, Kryger-Baggesen P, Hartvigsen J (2009) Retropharyngeal tendinitis may be more common than we think: a report on 45 cases seen in Danish chiropractic clinics. J Manipulative Physiol Ther 32(4):315-320

3. Park R, Halpert DE, Baer A, Kunar D, Holt PA (2009) Retropharyngeal calcific tendinitis: case report and review of the literature. Semin Arthritis Rheum 39(6):504-509 [Epub 2009 Jun 21]

4. Razon RV, Nasir A, Wu GS, Soliman M, Trilling J (2009) Retropharyngeal calcific tendonitis: report of two cases. J Am Board Fam Med 22(1):84-88

5. Chung T, Rebello R, Gooden EA (2005) Retropharyngeal calcific tendinitis: case report and review of literature. Emerg Radiol 11(6):375-380 [Epub 2005 Jul 15]
6. Jiménez S, Millán JM (2007) Calcific retropharyngeal tendinitis: a frequently missed diagnosis: case report. J Neurosurg Spine 6(1):77-80

7. Offiah CE, Hall E (2009) Acute calcific tendinitis of the longus colli muscle: spectrum of CT appearances and anatomical correlation. Br J Radiol 82(978):e117-e121

8. Harnier S, Kuhn J, Harzheim A, Bewermeyer H, Limmroth V (2008) Retropharyngeal tendinitis: a rare differential diagnosis of severe headaches and neck pain. Headache 48(1):158-161 [Epub 2007 Sep 12]

9. Omezzine SJ, Hafsa C, Lahmar I, Driss N, Hamza H (2008) Calcific tendinitis of the longus colli: diagnosis by CT. Joint Bone Spine 75(1):90-91

10. Bladt O, Vanhoenacker R, Bevernage C, Van Orshoven M, Van Hoe L, D'Haenens P (2008) Acute calcific prevertebral tendinitis. JBR-BTR 91(4):158-159

11. Fahlgren H (1986) Retropharyngeal tendinitis. Cephalalgia 6(3):169-174

12. Sanghvi DA, Jankharia BG, Purandare NC, Sundaram M (2006) Radiologic case study. Acute calcific retropharyngeal tendinitis. Orthopedics 29(7):561, 650-1

13. Oliva F, Giai Via A, Maffulli N (2012) Physiopathology of intratendinous calcific deposition. BMC Med 10(1):95

14. Rui YF, Lui PP, Chan LS, Chan KM, Fu SC, Li G (2011) Does erroneous differentiation of tendon-derived stem cells contribute to the pathogenesis of calcifying tendinopathy? Chin Med J (Engl) 124(4):606-610

15. Uhthoff HK, Loehr JW (1997) Calcific tendinopathy of the rotator cuff: pathogenesis, diagnosis, and management. J Am Acad Orthop Surg 5(4):183-191 